\title{
EDITORIAL
}

\section{Integrated Child Health and Development Program: A Key for Human Development}

In over a decade, Ethiopia has demonstrated a significant reduction in child mortality where it has achieved Millennium Development Goal (MDG)-4 two years ahead of the planned time. The Ethiopian government and the Federal Ministry of Health have taken encouraging steps by investing in holistic development and health care. There has been substantial progress on several health, economic and development indicators in Ethiopia. For the success in the health sector, Ethiopia pioneered a strong community health system and implemented integrated management of neonatal and childhood illnesses (IMNCI). Across the country, over 38,000 health extension workers have been deployed to provide basic preventive and curative services at community and household levels; many health facilities have been built and become operational. This quick expansion of rural health services and the implementation of IMNCI have laid the groundwork for a significant reduction in child mortality. This success has generated great benefits for the entire nation but the child's life above all. The experience and success also gave a great lesson to the rest of the world.

While celebrating the success of reducing child mortality, a strategy has to be designed to further reduce under-five children mortality and improve child health in general. Although survival of children has improved significantly in the past nearly two decades, many surviving children are not reaching their developmental potential for various reasons. A significant proportion of children are still undernourished, unimmunized, subjected to child abuse and lack development stimulatory options. This negatively affects their physical growth, education and productivity. Parents, community and religious leaders, child health practitioners and the government have responsibility for creating optimum environment for children to reach their maximum potential. If children are to develop to their potential, it is vital that their families and the society in which they are living understand their make-up and needs so that adults and children together can ensure that latter are properly nurtured. Without delay, our health system has to strengthen the integrated child health and development program that promotes holistic (physical, cognitive, emotional and social) health and wellbeing of children.

The current issue (Vol. 24, No 3), the third issue in the leap of EJHS to a quarterly publication, contains ten original articles on varied subjects and three case reports focusing on different cases of rarity. I invite readers to read through these articles and appreciate or utilize the contents. I also encourage readers to forward comments and suggestions.

Abraham Haileamlak (MD)

Editor-in-Chief 